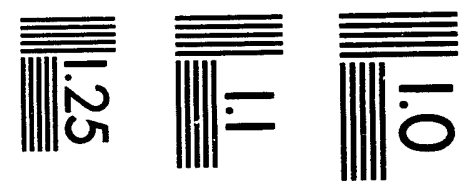

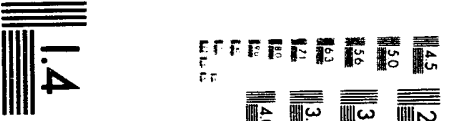

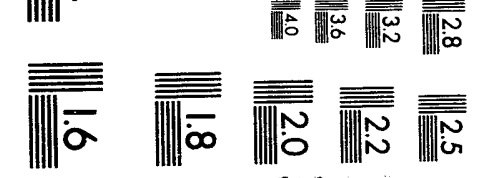



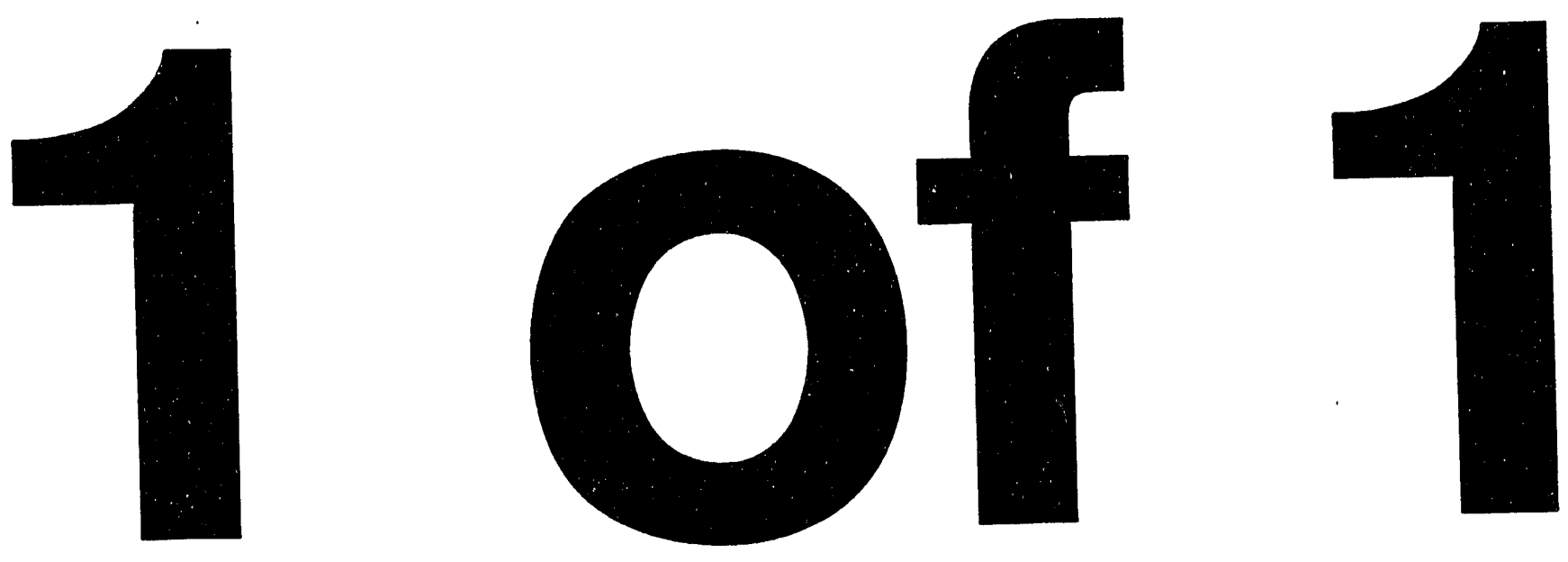


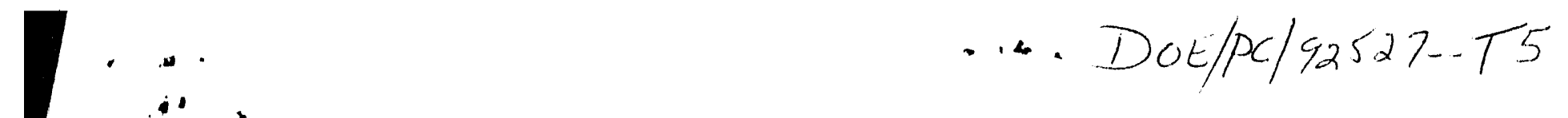

MAR 281994

\author{
AWARD NO. DE-FG22-92PC92527 \\ Quarterly Report No. 5 \\ Research Period: September 18, 1993 - December 17, 1993 \\ HINDERED DIFFUSION OF COAL LIQUIDS
}

Theodore T. Tsotsis, Co-Principal Investigator

Muhammad Sahimi, Co-Principal Investigator

University of Southern California

Department of Chemical Engineering

Los Angeles, California 90089-1211

Ian A. Webster, Co-Principal Investigator

UNOCAL Corporation

1201 West 5th Street

Los Angeles, California 90051

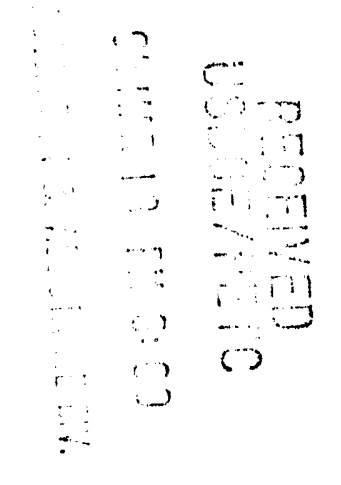

US/DOE Patent Clearance is not required prior to the publication of this document.

\title{
DISCLAIMER
}

\begin{abstract}
This report was prepared as an account of work sponsored by an agency of the United States Government. Neither the United States Government nor any agency thereof, nor any of their employees, makes any warranty, express or implied, or assumes any legal liability or responsibility for the accuracy, completeness, or usefulness of any information, apparatcs, product, or process disclosed, or represents that its use would not infringe privately owned rights. Reference herein to any specific commercial product, process, or service by trade name, trademark, manufacturer, or otherwise does not necessarily constitute or imply its endorsement, recommendation, or favoring by the United States Government or any agency thereof. The views and opinions of authors expressed herein do not necessarily state or reflect those of the United States Government or any agency thereof.
\end{abstract}


TABLE OF CONTENTS

INTRODUCTION

PROJECT DESCRIPTION 10

A. EXPERIMENTAL TASKS 11

A.1. Measuring Asphaltene Diffusivity in Model

Catalysts under Realistic Temperature

and Pressure Conditions

A.2. Measuring Asphaltene Diffusivities

in Sol-Gel Ceramic Membranes

A.3. Measuring Asphaltene Diffusivity in Model and Real Membranes under Reactive Conditions 12

B. THEORETICAL TASKS

B.1. Study of Hindered Transport in a Single Pore 13

B.2. Transport and Reaction in Networks of Interconnected Pores 14

B.3. Monte Carlo and Molecular Dynamics Simulations

B.3.1. Dilute Simulations $\quad 15$

B.3.2. Low Density Diffusion with Adsorption/Desorption $\quad 16$

B.3.3. Role of Intramolecular, Intermolecular and Surface Forces-Accounting for $\begin{array}{ll}\text { Aggregation and Delamination Phenomena } & 17\end{array}$

B.3.4. Molecular Dynamics Simulations 19 RESEARCH COMPLETED THIS QUARTER 20 LITERATURE REFERENCES APPENDIX A - Prepinta Rempacel 27 


\section{HINDERED DIFFUSION OF COAL LIQUIDS}

\section{INTRODUCTION}

The design of industrial catalysts requires that the diffusivity of the reacting species within the catalyst be accurately known. Nowhere is this more important than in the area of coal liquefaction and upgrading of coal liquids. In this area one is faced with the task of processing a number of heavy oils, containing metals and other contaminants, in a variety of process dependent solvents. It is important, therefore, on the basis of predicting catalyst activity, selectivity, and optimizing reactor performance, that the diffusivities of these oil species be accurately known.

Several studies exist [1], emphasizing the importance of diffusion in processes involving coal and petroleum liquids. Spry and Sawyer [2] measured the demetallization rates of a crude oil using various catalysts and found these rates to depend on average pore size, in agreement with their simple model, based on the theory of Anderson and Quinn [3]. Inoguchi [4] observed that optimum activity for desulfurization of heavy crudes occurs with catalysts with pore sizes of $100 \AA$ and for vanadium removal with pore sizes between 120 and $140 \AA$. Eigenson et al. [5] found increased catalytic activity for $\mathrm{MoO}_{3}$ on $\mathrm{Al}_{2} \mathrm{O}_{3}$, when the pore size increased from 70 to $150 \AA$. Significant intraparticle diffusion effects have been observed during asphaltene cracking and the desulfurization of asphaltenic and nonasphaltenic fractions of a residuum by Philippopoulos and Papayannakos [6]. Ternan and coworkers [7] have reported significant pore size effects during the catalytic processing of Athabasca bitumen. Studies have also been published on the importance of intraparticle diffusion during coal liquefaction and coal liquid upgrading, including those of Polinski and coworkers [8], Scooter and Crynes [9], and Yen et al. [10]. Workers at Amoco have found [11] that micropore volume in a critical pore size range, i.e. narrowly distributed about $120 \AA$ diameter pores, was very important in coal liquefaction catalyst activity. McCormick et al. [12] have shown during their hydrotreating studies that activities for both hydrogenation and dehydrogenation reactions are highly correlated (>99\% confidence) with the pore volume in the preferred range $(60-200 \AA)$ in diameter. The importance of catalyst average pore diameter and pore length during the hydrocracking of solvent refined coals and lignites has been discussed by Berg and Kim [13]. Theoretical studies have also been published, which incorporate hindered diffusion in order to predict optimal pore sizes during petroleum liquid upgrading [14] and catalytic coal liquefaction [15].

The molecules comprising coal liquids can range from less than 10 to several hundred $\AA$ in diameter. Their size is, therefore, comparable to the average pore size of most hydroprocessing catalysts. Thus, during processing, transport of these molecules into the catalyst occurs mainly by "configurational" or "hindered diffusion," which is the result of two phenomena occurring in 
the pores; the distribution of solute molecules in the pores is affected by the pores and the solute molecules experience an increased hydrodynamic drag. The field of hindered diffusion has been reviewed by Deen [16]. The earliest studies in the field were by Renkin et al. [17]. Based on the work of Ferry [18], they developed an expression to describe hindered diffusion

$$
\frac{D_{e}}{D_{b}}=(1-\lambda)^{2}\left(1-2.1044 \lambda+2.089 \lambda^{3}-0.948 \lambda^{5}\right),
$$

where $D_{e}$ is the effective "hindered" diffusivity, $D_{b}$ the bulk diffusivity and $\lambda$ the ratio of solute to pore radius. The first factor in this expression accounts for the partitioning effect, and the second for increased hydrodynamic drag in the pore. Anderson and Quinn [3] and Brenner and Gaydos [19] have theoretically verified these results in the region of small $\lambda$ s (their work is a generalization of Taylor-Aris dispersion theory [20] for spherical molecules with sizes comparable to the diameter of the cylindrical tube). A number of other theoretical studies have also been devoted to this problem [21]. Since the work of Renkin et al. [17], there have been a number of additional experimental studies of diffusion in restricted porous environments. Satterfield et al. [22] investigated hindered diffusion of binary systems of paraffins and aromatic hydrocarbons in silica alumina catalysts. They concluded that reduced diffusivity, in these systems, was only due to the hydrodynamic effects and their results indicate a linear dependence of $\ln \left(D_{e} / D_{b}\right)$ on $\lambda$.

Prasher et al. [23] also carried out studies using hydrocarbon solutes in aromatic and hydroparaffinic solvents. Contrary to Satterfield's results, they observed a solute partitioning effect dependent on the nature of the diffusing chemical species, rather than its molecular size, as one expects based on Renkin's theory. Colton et al. [24] studied diffusion of proteins and polystyrene in porous glass and found that protein diffusion follows Renkin's theory, while polystyrene behaves as a free draining molecule. Cannel and Rondelez [25] measured diffusion of polystyrene through a Nuclepore membrane and found that polystyrene diffusion follows Renkin's theory, if $\lambda$ is corrected by a factor of 1.45 . The above studies have been useful in showing that reduced diffusivities are observed in restricted environments. The pore systems utilized, however, were not well-defined and probably too complex to allow for fundamental questions to be answered. In the sixties, welldefined planar porous systems were developed. Price and Walker [26] demonstrated that uniform parallel pores of molecular dimensions can be formed, by a track etching process, in a number of alumino-silicate materials. Mica membranes prepared by this method were subsequently utilized in diffusivity measurements of nonhydrocarbon systems [27].

Studies of diffusion of petroleum and coal liquids are recent and few in number. In one of the earliest studies, Thrash and Pildes [28] studied the transport of asphaltenes derived from a Middle East high sulfur vacuum resid through mica membranes. The mica membranes had a pore diameter of $\sim 1200 \AA$. Thrash and Pildes claim that what they measure are bulk diffusivities from 
which they calculate Stokes-Einstein diameters of $16 \AA$. For the asphaltene concentration range of their experiments (50-500 ppm), they report no evidence of asphaltene molecular association. Shimura et al. [29] studied asphaltene diffusion in toluene through porous catalysts by uptaketype experiments. The bulk diffusivity of molecular weight (MW) fractionated petroleum pitches in dilute chloroform solutions was measured in a Northrup-McBain diaphragm cell by Sakai and coworkers [30]. Their studies led them to conclude that the pitch molecules examined are threedimensional structures with an oblate ellipsoidal shape, their equatorial diameter being $10-20 \AA$ and their axial ratio between 1 and 3. Jost et al. [31] also measured the bulk diffusivities of various oil residues in a THF/methanol solution by Taylor dispersion techniques. Recently, Mieville et al. [32] measured the diffusion of various asphaltenes, by uptake experiments, through catalysts with both unimodal and bimodal pore size distributions. In their experiments, Hondo asphaltene was found to have the highest diffusivity, which was attributed to its smaller average aromatic cluster.

Configurational diffusivity measurements of petroleum liquids, utilizing mica membranes, were first made by Baltus and Anderson [33] using a Wicke-Kallenbach-type diffusion cell to measure the diffusivity, through mica membranes, of asphaltenes derived from Kuwaiti atmospheric bottoms. They divided the overall MW range into five regions and assigned each to a "hypothetical" asphaltene fraction. Using GPC, they measured the diffusivity of each fraction for pore diameters ranging from 160 to $4400 \AA$. An experimental expression was found to relate the diffusivity $D_{e}$ of each fraction to its bulk diffusivity $D_{\infty}$, i.e. $D_{e} / D_{\infty}=\exp (-3.89 \lambda)$. Following the work of Baltus and Anderson, two other research groups have, in the last five years or so, focused their attention on the study of hindered transport of petroleum liquid macromolecules through restricted porous environments. Baltus and coworkers [34] have measured the diffusivity through polycarbonate and polyester membranes of asphaltenes isolated from Athabasca tar sand bitumen vacuum bottoms and a vacuum resid from a blend of Canadian crudes. They have also coupled the diffusivity measurements with intrinsic viscosity measurements of asphaltene solutions. Using models previously developed by Garcia de la Torre and Bloomfield [35], which represent the asphaltene in terms of assemblages of solid spheres, and by varying the size and number of such spheres, they were able to obtain a reasonable fit between their experimental results and theory.

Our group has also studied the diffusivity of petroleum derived asphaltenes [36]. We have investigated asphaltenes derived from a variety of California heavy crudes. Although our studies, in some aspects, paralleled those of Baltus and coworkers, our main objective was distinctly different from their objective. Voluminous literature exists on the detailed chemical structure of asphaltenes and other petroleum and coal liquid fractions like resins and oils. For asphaltenes, in particular, interest in their structure and reactive and transport properties has been steadily increasing among 
those working in the field of petroleum and coal liquid upgrading.

The term asphaltenes has been historically used to collectively describe the chemical components of a crude (or a coal liquid), which are generally more polar and larger in size. Since the earlier stages of the field of heavy oil upgrading, asphaltenes were defined as a solubility class of various components. Through the years, this definition attracted discussion and criticism (see, for example, Bunger and Cogswell [37]). Since some asphaltene properties are dependent on their isolation techniques, the relevant question here is whether or not the asphaltenes produced by such isolation techniques bear any resemblance and similarity to compounds found in the original crude, and whether or not the asphaltenes themselves exist in the original crude rather than simply being an artifact of their separation process. The debate over whether or not asphaltenes even exist, and if they do, how does one properly isolate and characterize them, has continued for years and will not be dealt with here. Comprehensive descriptions can be found in a number of papers [38]. The generally held view today is that asphaltenes do exist and are found in the original resid. One, however, cannot uniquely characterize an asphaltene (and, for that matter, any other resid fraction) based on a single property alone, such as solubility. For petroleum resids, two properties are needed to differentiate one resid fraction from the other. Which two properties one should use, however, still remains a mattor of debate. In his pioneering paper, Long [39] first suggested that the composition of petroleum liquids can be represented in terms of a plot of MW versus polarity. Long and Speight [39], furthermore, opted to use the solubility parameter as a measure of polarity and the $5 \%$ and $95 \%$ points of the MW distribution (as measured by GPC) as a measure of the MW. Wiehe [38], on the other hand, uses the number average MW (as measured by VPO) as a measure of MW and the average hydrogen content as a measure of the molecular attraction. For coal liquids, a measure of molecular attraction involving oxygen functionality would be required in addition to the average hydrogen content (in lieu of aromacity) and MW. This was the conclusion of Snape and Bartle [40] who found that three independent variables, i.e. number average MW, the proportion of internal aromatic carbon to total carbon and the percent acidic $\mathrm{OH}$ were needed to distinguish between benzene insolubles, asphaltenes and $n$-pentane solubles for coal-derived liquids.

Generally, the heavier the liquid one deals with, the larger the percentage of asphaltenes one finds in it. One of the obstacles in coal liquid (and resid) upgrading is the presence of heteroatoms, such as sulfur, nitrogen, oxygen and metals, which poison the catalysts used for hydroprocessing. In terms of composition, asphaltenes contain a greater percentage of heteroatoms than all other fractions and, therefore, present the greatest challenge during resid upgrading. Furthermore, most of the molecular components of an asphaltene are very large structures with sizes approaching that of a typical catalyst pore. Hydroprocessing of asphaltenes involves a number of parallel and/or 
consecutive reactions like hydrodesulfurization, hydrodenitrogenization, hydrodemetallation and cracking. Due to the large size of most asphaltene entities, all these reactions, as they occur within the catalyst pellets, are diffusion limited.

For many years, asphaltene and resid upgrading rector design has utilized the concept of asphaltenes being a single molecular entity, to which one can assign a unique effective diffusivity and other effective transport properties. This was one of the first issues we addressed in our studies of asphaltene diffusivity. Our experiments showed that the effective diffusivity of an asphaltene, as measured in a standard Wicke-Kallenbach cell using model porous membranes, varies as a function of experimental time by as much as an order of magnitude for the duration of an experimental run [36]. The diffusivity, furthermore, is a strong function of temperature and pressure, and whether the membrane is impregnated with a catalyst or not. One cannot, therefore, assign a unique experimental diffusivity value to an asphaltene molecule. Our experimental findings, though new, were surprising neither to us nor to those intimately involved in research on asphaltene chemical structure and properties. Asphaltenes are not uniquely defined simple molecular entities and generic structures, but rather a complex mixture of colloidal entities (micelles) of various sizes and shapes, consisting of assemblages of smaller particles, which in turn result from the clustering of lower MW components, all in a state of dynamic exchange strongly affected by the presence and polarity of solvents, temperature, pressure and fluid mechanical conditions [36, 41].

Our studies subsequently focused on the issue of whether the picture of an asphaltene "molecule" as described above, which emerges from the available chemical information, i.e., a dynamic system in a constant state of interchange is consistent with the experimental findings of diffusivity and transport of asphaltenes through model porous membranes. An experimental plan was devised, as outlined in Fig 1 to test these concepts. The asphaltenes used were $n$-pentane insolubles extracted from $\mathrm{H}$. „do Crude, a California outer continental shelf oil, furnished by UNOCAL. The diffusion cell was a stainless steel Wicke-Kallenbach-type apparatus consisting of two wellmixed compartments (half cells). During experiments of asphaltene diffusion, the half-cells were separated by a polycarbonate membrane containing straight, parallel, non-intersecting, cylindrical pores (supplied by Nuclepore Corp., Pleasanton, California). The membrane pore size varied between $100 \AA$ and $4000 \AA$. (Further details about our experimental system and techniques can be found elsewhere [36]. The first experiment was conducted with a $100 \AA$ membrane. We started with a 5 weight percent solution of asphaltene in xylenes, which was loaded in one of the half-cells, hereinafter referred to as the high concentration side (HCS). Pure xylenes were loaded in the other half-cell, the low concentration side (LCS). The asphaltenes were allowed to diffuse through the membrane for a predetermined period of time. During the experiment, samples were withdrawn 


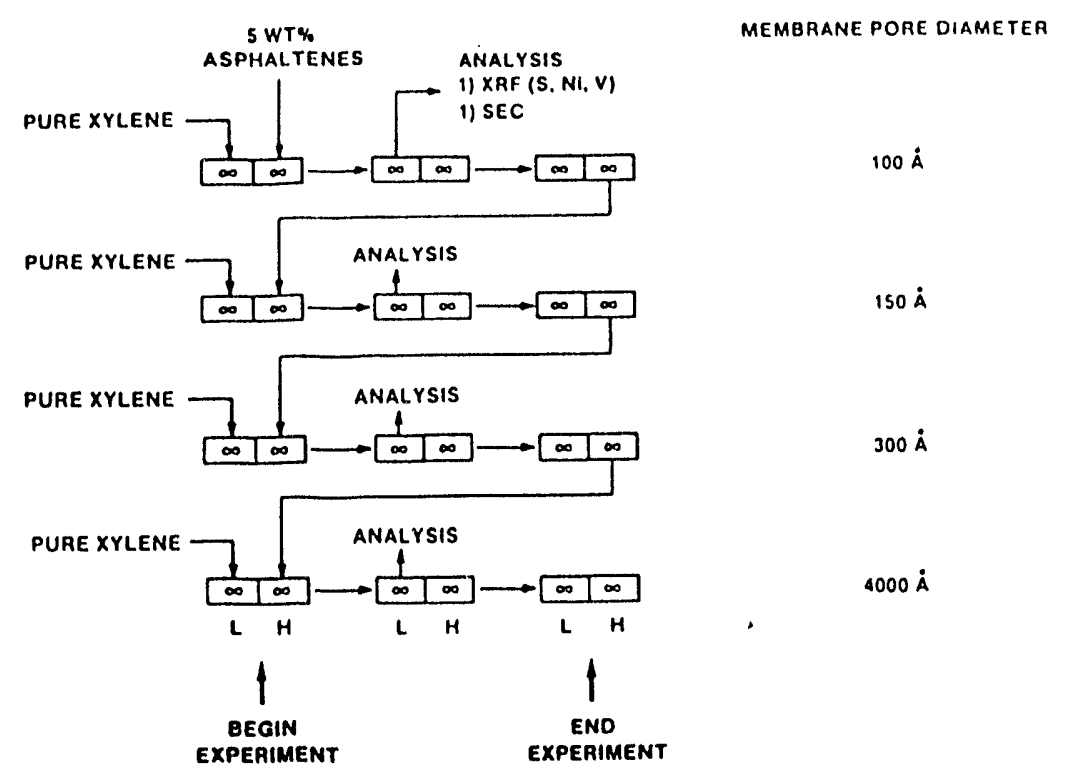

Fig.1. Experimental plan

from the LCS and analyzed for sulfur, nickel and vanadium concentrations by X-ray Fluoresence (XRF), and for MW distributions by Size Exclusion Chromatography (SEC), MW distribution of the various metal containing components by SEC/ICP and for overall asphaltene content. After the first experiment ended the second experiment was initiated with a $150 \AA$ membrane. Again, pure xylenes were loaded in the LCS. In this experiment, however, the HCS was filled with the asphaltene solution remaining in the HCS at the end of the first experiment with the $100 \AA$ membrane. Further experiments were done with the rest of the membranes starting again with pure xylenes in the LCSs and the HCSs containing the HCS solution carried over from the preceding experiment.

Some of the data collected from the diffusion experiments, depicting nickel and vanadium concentrations in the LCS as a function of run time are plotted in Figs. 2 and 3. Figs. 4 and 5 show typical SEC analyses of the LCS samples at various experimental times for two different pore sizes. One can draw the following conclusions from these figures (and the rest of the experimental data). First note Figs. 4 and 5, which show SEC analyses of LCS samples withdrawn at different run times. The polydisperse nature of asphaltenes is clear from these figures. Asphaltenes are not simple and uniquely defined molecules but rather consist of a whole range of molecular components of various sizes. The components with smaller sizes diffuse faster than the components with larger sizes and as a result the MW distribution of the asphaltene in the LCS at smaller times is skewed towards the lower MWs. At larger times, the MW distribution of the asphaltene in the LCS approaches that of the asphaltenes initially loaded in the HCS.

This, obviously, is an oversimplified picture of how asphaltenes diffuse through porous mem- 


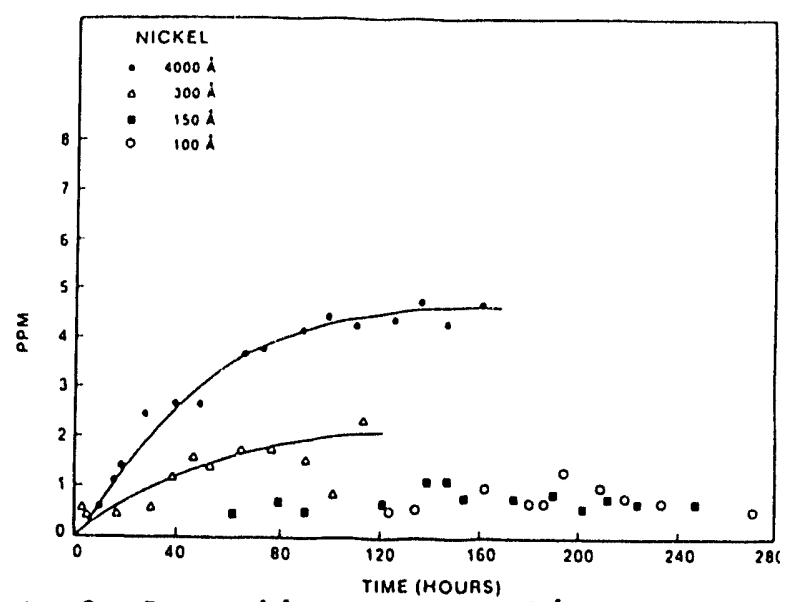

Fig.2. Low side concentration

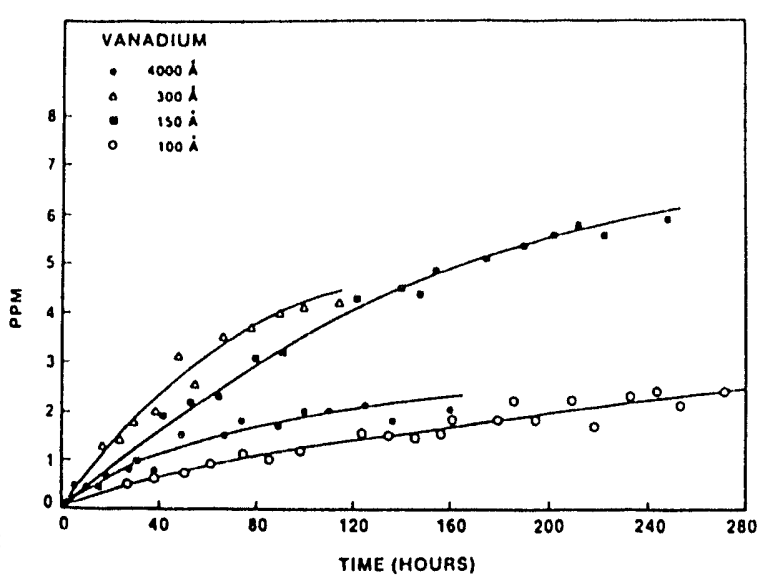

Fig.3. Low side Concentration.

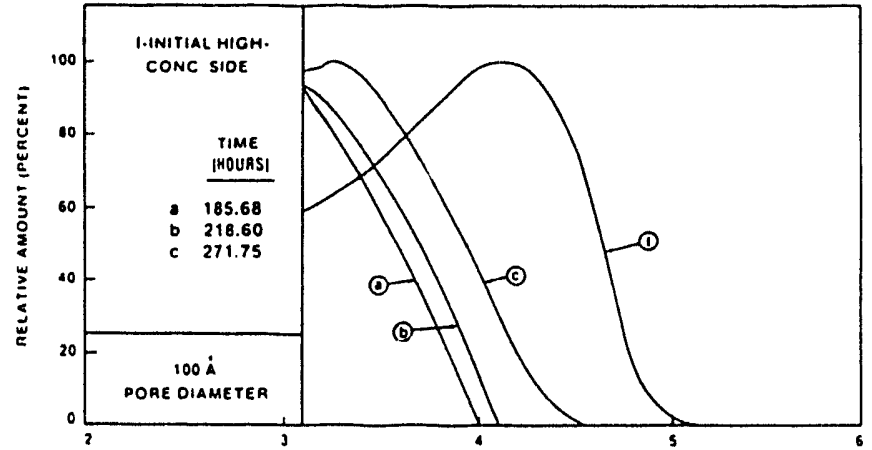

LOG MOLECULAR WEIGHT

Fig.4. Log Molecular weight

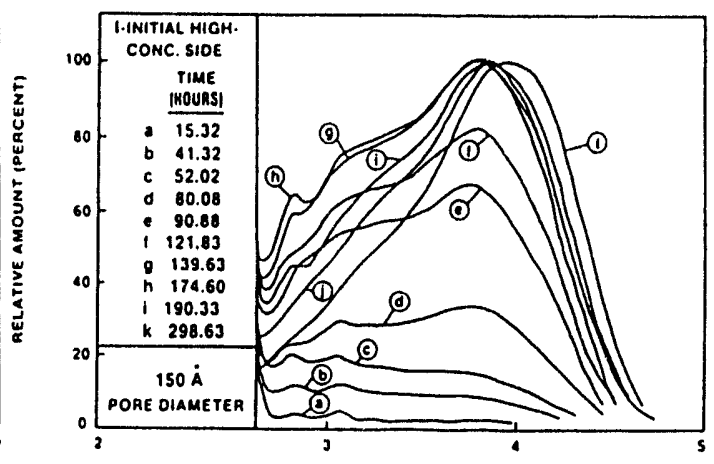

LOG MOLECULAA WEIGHT

Fig.5. Log Molecular weight
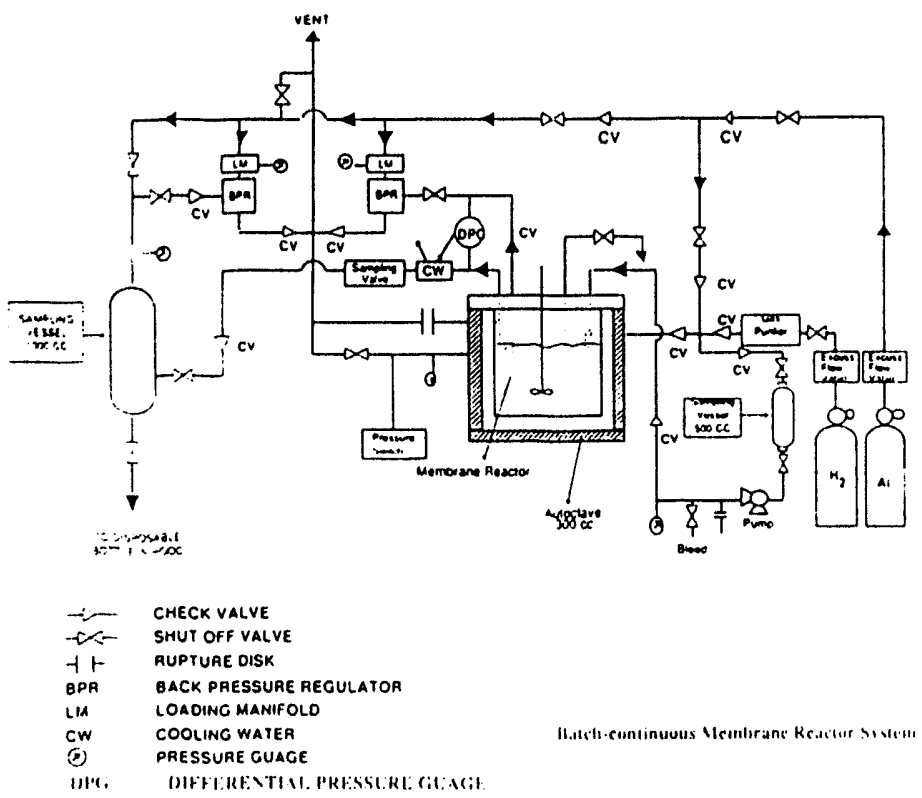

Fig.6. High temperature and pressure diffusion system. 


\begin{tabular}{|l||l|l|l|l||}
\hline $\begin{array}{l}\text { Nominal } \\
\text { Pore Size }\end{array}$ & Asphaliene & Sulfur & Nickel & Vanadium \\
\hline $100 \mathrm{~A}$ & - & 12.7 & 10.2 & 9.9 \\
\hline $150 \mathrm{~A}$ & 8.7 & 8.6 & 10.0 & 9.6 \\
\hline $300 \mathrm{~A}$ & 8.2 & 8.1 & 6.7 & 8.6 \\
\hline $500 \mathrm{~A}$ & 6.4 & 7.2 & 6.3 & 7.6 \\
\hline $1000 \mathrm{~A}$ & 4.6 & 5.7 & 5.5 & 6.5 \\
\hline
\end{tabular}

Table 1: Activacion energies in kcal/mole calculated from effective diffusivities.

branes. This mechanism of asphaltene transport is only consistent with the polydisperse nature of asphaltenes. On the other hand, any polydisperse polymer would diffuse in a similar fashion, as indicated in Figs. 4 and 5. To realize that asphaltenes diffuse in a uniquely different fashion, one has to examine Figs. 4 and 5 in conjunction with Figs. 2 and 3. Note, for example, Fig. 2. Clearly there are components in the initial asphaltene that are larger than $150 \AA$. In fact, out of the total nickel diffused throughout the whole series of experiments, only approximately $20 \%$ permeates, in the time allotted, through the 100 and $150 \AA$ membranes. How could it then be possible that the asphaltene at large times in Fig. 5 closely resembles the asphaltene initially loaded in the HCS? The only asphaltene structure capable of describing the data in Fig. 5 is the one we have previously described, namely that "the asphaltenes are not simple, single generic species but rather complex mixtures of micelles of various sizes and shapes, of unformed micelles, of small particles and of low MW components and attached alkyl chains, all in a state of dynamic exchange strongly affected by the presence of solvents, temperature and pressure and fluid mechanical conditions." Only a dynamic association-disassociation process can explain the fact that the large components of the asphaltenes, with nominal sizes larger than the diameter of the pore find their way from the HCS to the LCS.

The dynamic nature of the asphaltene molecule manifests itself in the effect of temperature and concentration on its transport. Table 1, for example, lists the activation energies calculated from the diffusivity data from heptane-insoluble asphaltenes at various temperatures. Activation energies for diffusion are generally of the order of $1 \mathrm{Kcal} / \mathrm{mole}$. The values in Table 1 are more typical of the association energies for individual asphaltene sheets reported by Dickie and Yen [41], typically 14 to $20 \mathrm{Kcal} / \mathrm{mole}$, rather than diffusion activation energies. The trends in terms of the pore size can be understood on the basis that asphaltene dissociation is expected to affect more strongly the transport through more constricted pores. The simplest model of an asphaltene molecule, which accounts for both its polydisperse character and its dynamic nature will faithfully reproduce the behavior in Table 1 .

There is obviously a lot more information included in Figs. 2-5 and similar such figures. For example, the mechanism of heteroatom transport emerging for these data is consistent with the 
detailed chemical information as to where these heteroatoms lie in the original asphaltene micelles and particles. More detailed such discussions can be found in the original publications [36]. Of more importance, however, are the implications of the transport mechanism for petroleum and coal asphaltenes that is emerging on resid and coal liquid upgrading reactor design. In our opinion, the implications are many and significant. Traditionally, ungrading reactor design has utilized the concept of overall effective transport properties for asphaltenes and other oil fractions. In view of the existing chemical information on the structure of asphaltenes, the practice has been characterized as simplistic but of some engineering utility. Our thesis in this proposal is that the practice is outright wrong and can lead to erroneous design calculations. For one thing, such effective properties cannot be measured (one order of magnitude variation in effective diffusivity was observed in some of our experiments). More elaborate techniques, furthermore, must be devised to relate diffusivities measured in a Wicke-Kallenbach cell or by uptake experiments to transport properties of relevance under reactive conditions.

Is the previously stated concept of the structure of an asphaltene, i.e., of an asphaltene being "a complex mixture in a state of dynamic exchange" significant for upgrading catalyst design? In our opinion, it is, because it offers plausible explanations for what is happening during upgrading. Look at Figs. 2 and 3. Most of the nickel, for example, found in a petroleum asphaltene should not even be penetrating the structure of a typical upgrading catalyst, if it was not for the concept of "dynamic exchange" between the various asphaltene components. The other plausible reason why typical upgrading catalysts can process the heteroatoms found in resids or coal liquids is that the structure of an asphaltene represented by the above model, although valid at low temperatures, will not survive the higher upgrading temperature and pressure conditions, i.e., the micelles and even the particles will break apart. Superficially, this explanation is in contrast with experimental data (like those, for example, by Wiehe [38] and our group [36]), which show that thermal treatment at upgrading conditions of asphaltenes does not completely destroy the asphaltenes, but instead results in their incomplete conversion to both lower MW fractions, like volatiles, saturates, aromatics and resins but also to higher MW components like coke. In our opinion, these data are significant but not necessarily conclusive about the structure of asphaltenes at higher temperatures and pressures in view of the asphaltenes being considered as "complex mixtures in a state of dynamic exchange." Assuming, on the other hand, that the asphaltene structure does not survive thermal treatment at upgrading conditions, what then is the purpose of studying such structures? In our opinion, the issue here is one of rates. Even if one is to assume that at elevated temperatures and pressures "micelles" and even "particles" break apart, the question to be raised is at which rate does this breakdown process occur? Contrary to laboratory reactors, where most of the studies of 
asphaltene's chemical structure have taken place, most industrial reactors are continuous systems. The state of the asphaltene "molecule" therefore does not only depend on the temperature, pressure and polarity of the solvent but also on the reactor's residence time. It is, therefore, very important to have a correct concept of the asphaltene's structure and through careful experimentation, one can then decide whether such a concept has any practical implications at realistic upgrading conditions.

It is the purpose of the project described here to provide such a correct concept of coal asphaltenes by careful and detailed investigations of asphaltene transport through porous systems under realistic process temperature and pressure conditions. The experimental studies will be coupled with detailed, in-depth statistical and molecular dynamics models intended to provide a fundamental understanding of the overall transport mechanisms.

\section{PROJECT DESCRIPTION}

Throughout the experimental runs described below we will utilize a high pressure, high temperature diffusion cell system, a schematic of which is shown in Fig. 6. This diffusion system has been tested through the measurement of the diffusivity of a number of model coal liquids.

The heart of the experimental system is a high pressure autoclave, which in its interior can accommodate one or several ceramic membranes. One side of these membranes is exposed to the contents of the autoclave, while the other side, through an independent flow system, is exposed to flowing pure solvent. The pressure in the interior and exterior of the membranes can be independently adjusted and controlled. This is also true with the flow rate of the solvent in the interior of the membrane. The diffusion experiments are initiated by placing the coal liquid solution (model liquids or asphaltenes) in the autoclave space exterior of the membrane, pressurizing the exterior and interior membrane volumes and initiating the flow of the solvent. One has the option of running the experiment in a batch (exterior)-continuous (interior) or batch-batch mode. The option also exists for loading catalyst in the exterior volume either in a pellet or slurry form or using metal impregnated membranes for simultaneously studying transport and reaction. Model membrane preparation and characterization will be carried out both at USC and under the direction of one of us (I. A. Webster) at the UNOCAL Science and Technology Division, of UNOCAL Corporation (USTD). UNOCAL, in addition, will contribute technician and machine time on apparatuses, such as Auger and XPS, preparative GPC, SEC, XRF, SEC/ICP, Low Angle Light Scattering Photometer, Electron Microscope, Atomic Adsorption, Porosimeters and BET.

The project is of both experimental and theoretical nature and is divided into a number of tasks, a brief description of which follows. 


\section{A. Experimental Tasks}

\section{A.1. Measuring Asphaltene Diffusivity in Model Catalysts under Realistic Tempera- ture and Pressure Conditions}

The asphaltenes will be isolated from coal liquids provided by PETC using a continuous type asphaltene isolation apparatus. This is a UNOCAL proprietary asphaltene isolation apparatus, a replica of which has been constructed at USC. Both pentane and heptane insoluble asphaltenes will be isolated.

The model membranes will be polymeric membranes, but also anodic aluminas, which have straight, non-intersecting pores. The anodic aluminas are produced by direct electrochemical anodization of aluminum sheets [42]. The anodization technique allows [43] for control of pore size, structure and morphology of the $\mathrm{Al}_{2} \mathrm{O}_{3}$ films formed, which depend on parameters such as voltage, temperature, current density, $\mathrm{pH}$ and type of electrolyte used. Parallel pores of uniform size (15$2000 \AA$ ) have been formed with pore densities as high as $10^{11}$ pores $/ \mathrm{cm}^{2}$. Our current anodization cell is based on a design by Itaya et al. [43] and allows for in situ anodization and barrier film removal (further details can be found in some of our recent publications [42]). During barrier film dissolution the porous alumina film is protected by maintaining an immiscible fluid head on the porous film side.

The experimental technique to be followed has already been described above. The asphaltene solution will be loaded in the autoclave in the space exterior to the membrane and pressurized under $\mathrm{H}_{2}$ or mixtures of $\mathrm{H}_{2}$ in $\mathrm{Ar}$ (see below). The interior and exterior membrane volumes will be sampled periodically and analyzed by techniques such as XRF for heteroatom analysis, SEC for MW distribution, SEC/ICP for heteroatom specific MW distribution, overall asphaltene content, NMR and FTIR for overall functional group identification and by Gas Chromatography. Parameters to be investigated include (but are not limited to): (1) The effect of asphaltene concentration on the overall transport mechanism; (2) The effect of temperature and overall pressure and pressure gradient across the membrane (the pressure of the interior and exterior membrane volumes can be independently adjusted and controlled); (3) The effect of solvent and type of asphaltene (i.e., pentane or heptane insoluble, type of coal liquid it came from); and (4) The effect of pore size (the anodic membranes have unimodal straight non-intersecting pores) and membrane surface morphological properties, which can be varied by the choice of electrolyte and post-anodization treatments.

When these experiments are completed a better picture, we hope, will emerge of the mechanism of coal asphaltene transport under realistic temperature and pressure conditions. 


\section{A.2. Measuring Asphaltene Diffusivities in Sol-Gel Ceramic Membranes}

Anodic membranes have unimodal straight, non-intersecting pores. As such, they allow for unique insight into the mechanism of the transport processes occurring since one does not have to worry about the complications resulting from the tortuous porous structure and to account for an adjustable parameter, i.e., the tortuosity factor. On the other hand anodic membranes are somewhat poor analogs of the true hydroprocessing catalysts, which have a complex tortuous porous structure.

Recently developed Sol-Gel type $\gamma-\mathrm{Al}_{2} \mathrm{O}_{3}$ membranes offer a compromise and a middle ground between the ideal structure of anodic aluminas and the complex structure of true upgrading catalysts. These membranes also have an extremely narrow unimodal pore size distribution (like the anodic aluminas) but have tortuous porous structures. Through their use we hope to gain an additional insight into the complications in the transport mechanisms of asphaltenes arising from the tortuosity of the porous medium.

The Sol-Gel membranes we will utilize will be Membralox ${ }^{\mathrm{TM}}$ asymmetric alumina membranes, which have been previously utilized by our group in catalytic membrane reactor investigations [44]. The minimum pore size of these membranes is $40 \AA$.

\section{A.3. Measuring Asphaltene Diffusivity in Model and Real Membranes under Reactive Conditions}

The issue of whether the intraparticle diffusivity measured under reaction conditions is the same with that measured under non-reactive conditions is of fundamental interest and the subject of ongoing debate [45-47]. For example, Otani and Smith [45] reported the effective diffusivity under reaction conditions to be only about $20 \%$ of that measured in the absence of reaction. In contrast Ryan et al. [46] have theoretically argued that the effective diffusivity should be independent of the chemical reaction rate. We have also shown theoretically [47] that, as is also true with facilitated transport through biological and polymeric membranes [48], in the presence of surface reactions with fast and slow steps involving mobile surface species, diffusivities measured under non-reactive conditions bear no similarity or relevance to diffusivities prevailing under reactive conditions. Under such conditions, of course, even the strict applicability of classical transport reaction equations is in doubt [46-50]. The asphaltene, due to the action of solvents and its interaction with other asphaltene molecules and the solid porous walls, is constantly fragmenting and agglomerating. In the absence of a porous barrier one would expect an asphaltene solution to reach a state of pseudo-dynamic equilibrium. When a porous barrier is present, however, as in the case with our experiments, this dynamic equilibrium is shifted towards lower MW fragments, since these fragments preferentially leak out of the membrane, i.e., one attains a form of facilitated fragmentation. In a real catalyst 
particle the situation, of course, is very similar. One does not have a LCS, where the low MW fragments will diffuse to but the low MW fragments are removed by the chemical reaction, i.e., again a chromatographic separation of the asphaltene occurs as it moves from the bulk solution into the particle's center.

These are the types of questions and issues we hope to address in this task. Our experimental system is very flexible and allows for the upgrading catalyst to be placed in the exterior membrane volume in the form of pellets in a small rotating basket, to be slurried in the asphaltene solution or in the solvent circulating in the interior membrane volume. The catalytically active component to be utilized will be Mo promoted with either Co or Ni. Additional parameters to be investigated (beyond those of Tasks I and II) are the amount of metal present and the effect of $\mathrm{H}_{2}$ partial pressure, by using mixtures of $\mathrm{H}_{2}$ and Ar.

\section{B. THEORETICAL TASKS}

\section{B.1. Study of Hindered Transport in a Single Pore}

As mentioned in the Introduction, a number of theoretical studies have been devoted to this problem. Starting with the comprehensive works of Brenner and coworkers [19], Anderson and Quinn [3] and Paine and Scherr [51]. The work of Brenner et al. and Paine and Scherr is a generalization of the Taylor-Aris [20] dispersion theory for spherical molecules with sizes comparable to the diameter of the cylindrical tube, in which hindered transport takes place. These and subsequent studies on hindered diffusion have not considered the case of simultaneous transport and reaction/adsorption in the pores.

As the first task of our theoretical work, we intend to study hindered transport of non-spherical molecules in cylindrical pores. The molecules comprising coal liquids have much more complex structures and geometries than the molecules studied in the field of hindered diffusion, where even the case of an ellipsoidal molecule in a cylindrical pore has not been completely analyzed (though some progress has been made by Brenner and coworkers [19]). Furthermore, little is known on the transport of large molecules in pores (cylindrical or not) in which a reaction is also taking place. We intend to study such problems by generalizing the approach of Brenner and coworkers, which is a statistical method, by which one calculates the probability density function for the center of the molecule to be at a position $r$ at time $t$. From the second moment of this function, $\left\langle r^{2}\right\rangle=6 D_{e} t$, the effective diffusion coefficient is determined. The results of this part of our study will be essential to the next stage of our investigation described below. Due to the inherent complexity of the approach, there is an upper limit on the degree of structural and geometric complexity of the molecules that can be studied by this technique. More complex molecular shapes, conceivably more consistent with our understanding of asphaltene molecules, will be employed in the Monte Carlo simulations 
described below. The value of the techniques of Brenner and coworkers lies in their simplicity of application, in that they do not require significant numerical computations.

\section{B.2. Transport and Reaction in Networks of Interconnected Pores}

As the second task of our work, we shall study hindered transport, reaction and adsorption in three-dimensional networks of interconnected pores. Any porous system can be mapped onto [50] a random network of bonds (pore throats) connecting to each other at sites (pore bodies) of the network. The resulting network usually has a random topology. The geometrical properties of the porous medium can be incorporated into the structure of the network by assigning randomlyselected effective radii to the bonds of the network. These can be chosen from a realistic pore size distribution obtained from, e.g., porosimetry. The bonds can, in principle, take on any shape, e.g., cylindrical tubes, sinusoidal or converging-diverging channels, or constricted tubes. Our networks will be constructed to simulate our Sol-Gel ceramic membranes used in our experiments.

By modeling the porous medium as a network of interconnected pores, one can generalize the work of Brenner and coworkers [19] to describe hindered transport and reaction/adsorption in complex porous systems. At the pore level, hindered transport of molecules is governed by a convective-diffusion equation (CDE) or its generalization obtained in Task B.1. One then needs to describe how transition of molecules from one pore to the next takes place. A rigorous approach is to write down a mass balance for a $Z$-coordinated pore body (node) $i$,

$$
\sum_{j}^{Z} S_{i j} J_{i j}=0
$$

where $S_{i j}$ is the effective cross section area of the pore throat between pore bodies $i$ and $j$ and $J_{i j}$ the flux in that pore. If this equation is written for every node of the network, one will obtain a set of simultaneous equations for the concentration of the diffusing molecules throughout the entire network. From the solution, one can then determine the effective transport properties of the molecules. This approach necessitates the use of large scale Monte Carlo computer simulations to calculate the quantities of interest.

Since the description of transport at the pore level is rigorous (see Task B.1), our estimates of the transport properties for the entire network will also be rigorous and exact, provided that the network model closely resembles the actual porous medium. This task has recently been initiated, and the preliminary results are encouraging [52].

\section{B.3. Monte Carlo and Molecular Dynamics Simulations}

Transport and reaction of molecules like asphaltenes in porous media is a complex process, in which various interacting factors may all be significant. These include: (a) The structure of the porous medium; (b) the molecular nature of the diffusing species, their shape, size and molecular 
flexibility; (c) the intermolecular interactions between the diffusing molecules as well as their interaction with the porous media; (d) the adsorption and desorption of the molecular species on the surface of the pores; and (e) the reaction on the pore surface, including the possible role of surface diffusion.

Given the complexities of the problem, computer simulation is the ideal technique to conduct such studies. In Tasks B.1 and B.2 we described simulation methods for solving such problems. These methods are restricted, however, to dilute systems, i.e., those in which only a few molecules diffuse and react in the system, whereas in reality this may not be the case. If the concentration of the diffusing species is high, then, intermolecular interactions, and also the interaction between the molecules and the pore surface become very important. Moreover, the approaches described in Tasks B.1 and B.2 can only be implemented for relatively simple molecular shapes. Asphaltenes, on the other hand, have extremely complex shapes. Since only limited detailed experimental data on the chemical reactivity of asphaltenes exist in the literature at the present time, for the purposes of this project, we will only concentrate our attention on understanding the physical characteristics of, and the physical processes taking place, namely, the diffusion, adsorption and desorption of molecular species in the catalyst particle and the dependence of these processes on the structure of the pore space and the nature of the molecules. Our tools are Monte Carlo and Molecular Dynamics simulations, which can naturally take into account the effect of every important factor. In what follows we outline our research plan for a comprehensive, step by step, theoretical and computer simulation studies of these phenomena.

\section{B.3.1. Dilute Simulations}

In order to understand the role that the porous medium's structure and the intermolecular and surface forces play, we will first consider the case of non-interacting molecules. This corresponds to dilute concentration experiments.

The appropriate structure, e.g., a single pore or a network of interconnected pores, will be modelled depending on whether we simulate our experimental anodic or Sol-Gel membranes. After generating the pore structure of the system to initiate the computer simulations, $N$ molecules will be injected into the pore space; this is time $t=0$. The molecular shapes used will be consistent with our understanding of asphalthene molecules (see Introduction/Experimental section) since in a simulation of the kind described here, any molecular shape and MW distribution can be used without much difficulty. Each molecule will perform a random walk (Brownian motion) in the pore space representing the diffusion process. At every step of the simulation, a molecule will select one of the available directions, with an equal probability, to make a transition to another part of the pore space. The maximum length of the step is held constant determined by an acceptance 
rate of $50 \%$. Each time a direction is selected, the molecule (i.e., its center) is moved to the new position. In the low density limit, a molecule does not meet another so there is no excluded volume except for that associated with the pore surface. This type of simulation can be implemented in two ways. One can inject into the system one molecule at a time, follow its motion and compile the statistics of interest. Alternately, one can inject simultaneously $N$ molecules intc the system. Each of the $N$ molecules are then displaced in turn as described above for the sequential injection method. For sufficiently large $N$, the results are independent of $N$. The simultaneous injection of many molecules implementation is more efficient when parallel processing is available. While use of random walks for simulating diffusion is not a new idea, to the best of our knowledge, aside from our recent work [53], no one has used this technique to simulate diffusion of large and complex molecules of various shapes in a restricted pore spaces such as those considered here.

For this series of initial simulations, we do not permit adsorption or reaction on the pore surface, to be accounted for in the next series of simulations (see below). The effective diffusivity of the molecules can be evaluated by determining the time dependence of the mean-squared displacement of the center of mass of the molecules

$$
r^{2}=\left\langle\left[r_{c m}(t=0)-r_{c m}(t)\right]^{2}\right\rangle
$$

where $\langle\cdot$.$\rangle denotes an average over all molecules and their initial positions in the system. In$ the limit of long times and for large systems, the ratio $r^{2}(t) / t$ is proportional to the $D_{e}$ of the molecules. (The above calculations are, of course, repeated for a large number of time steps and many randomly-selected initial positions of the molecules, and the averages of the quantities of interest are computed, in order to obtain representative values of effective diffusivities.) A series of simulations for pore size distributions, and various molecular shapes are planned to probe the effect of the pore structure on this very simple diffusion process. The key variable here will be the relative sizes of the molecule and the pores, and the key question will be whether, consistent with the experimental observations, $D_{e}$ will eventually reach a constant value.

\section{B.3.2. Low Density Diffusion with Adsorption/Desorption}

In this phase of the work, we will look at the effect of adsorption and desorption. We will first consider the case of irreversible adsorption. In the simulation, when a molecule comes in contact with a pore surface, it will be adsorbed with probability $p$ which is proportional to a Boltzman factor $\exp \left(-c \epsilon_{i}\right), c$ being a constant and $\epsilon_{i}$ the surface binding energy. $p \rightarrow 0$ describes a system under sorption kinetic control, whereas $p \rightarrow 1$ represents a diffusion-controlled process. An adsorbed molecule results in an excluded surface and volume in the pore space. The simulations will be terminated when a large number of molecules have been adsorbed on the pore surfaces so that 
there is no significant additionial adsorption, or the adsorbed molecules, due to their finite volumes, have effectively blocked the pore space and no further macroscopic molecular motion occurs. By this simulation procedure, termed the Dynamic Monte Carlo Method (DMCM), one can calculate all the dynamic, and static properties of interest.

Next to be considered is the case of reversible adsorption. The problem is more complex since now one must consider the kinetics of the adsorption/desorption process in addition to diffusion. The injection rate of molecules must be controlled in a physically realistic manner. We believe that it is physically plausible to assume that a state of equilibrium exists between the molecules in the immediate surrounding of the porous medium and those on its outer periphery. This means that a certain fraction, say $f$, of the layer of the pore space is occupied by diffusing molecules. Throughout the simulation, $f$ is maintained constant, which means that as soon as a molecule leaves one of the peripheral positions, another molecule must be introduced into one of the vacant peripheral cells, but not necessarily the one just vacated. The relative time scales of the diffusion, adsorption and desorption processes can be varied by changing the adsorption probability $p$ and the ratio $\alpha=$ desorption rate/adsorption rate. The limit $\alpha \rightarrow 0$ corresponds to irreversible adsorption. Setting $\alpha=1$ will require each molecule to remain on the surface for a fixed period of time (say $m$ time steps) before they are eligible for desorption. When interpreting the results several factors must be considered. In case of the diffusion-limited regime, sorption will primarily occur at the outside external perimeter of the catalyst particle and the phenomenon of poremouth blocking will occur. The effect of molecular sorption on diffusivity is then a strong function of the adsorption/desorption rates relative to the diffusive fluxes. When diffusion is not rate limiting, a large number of molecules are adsorbed on the pore surfaces. Even then, however, pore plugging can occur but the phenomenon is a percolation process. The DMCM technique to be developed in this Task can be used to systematically study the effect of all system parameters on the transport process.

\section{B.3.3. The Role of Intramolecular, Intermolecular and Surface Forces - Accounting for Aggregation and Delamination Phenomena}

Intramolecular and intermolecular forces determine the size, shape and flexibility of the diffusing molecules and the attraction/repulsion between molecular pairs. The number of possible parameters can be very large, especially for polydisperse complex molecules like asphaltenes and cannot be determined a priori in our simulations due to the limited understanding of intermolecular and surface forces. We will therefore start by studying the diffusion and adsorption of molecules with relatively simple shapes.

The simulations are started by generating a molecular analog of the asphaltene's structure. One 
then has to account for the intermolecular forces that play an important role in the diffusion of the molecules. But what is the appropriate expression for the intermolecular forces? In most previous MC simulations of diffusion processes, a Lennard-Jones (LJ) potential has been used, i.e.

$$
\phi_{i j}=4 \epsilon_{i j}\left[\left(\frac{\sigma_{i j}}{r}\right)^{12}-\left(\frac{\sigma_{i j}}{r}\right)^{6}\right]
$$

where $\phi_{i j}$ is the potential energy experienced by the $i-j$ pair of molecules $\epsilon_{i j}$ and $\sigma_{i j}$ are the energy and size parameter and $r$ the intermolecular separation. The $\mathrm{J}, \mathrm{J}$ potential, most likely is too simple to account for the intermolecular forces between complex molecules. To resolve this problem, one can treat $\epsilon_{i j}$ and $\sigma_{i j}$ as adjustable parameters such that the simulation would reproduce one set of data (e.g., at a given $T$ and $P$ ) and, then, use the resulting values to study the system under a different set of conditions. This procedure has recently been used by several authors with a reasonable degree of success. At the next level of sophistication are site-site potentials and other more complex-forms of intermolecular forces [54] that have been quite successful. These will also be employed in our simulations. The molecule-wall interactions can similarly be modeled using the following potential

$$
\psi(z)=\frac{8 \pi \epsilon}{5}\left[\frac{2}{5}\left(\frac{\sigma}{z}\right)^{10}-\left(\frac{\sigma}{z}\right)^{4}\right],
$$

where $\psi$ is the potential energy due to surface-molecule interactions, and $z$ the distance between the surface and the molecule's center of mass. Of course, because of the complex structure of asphaltene molecules, one can only use effective values of these parameters.

Different $\epsilon$ will be used to study the effect of the attraction of the pore surfaces. We start the simulation by placing molecules in a manner dictated by our experiments. All molecules in the system will then be given sequential random displacements in the normal Monte Carlo way, each displacement accepted with probability:

$$
p_{\text {displacement }}=\min [1, \exp (-c \Delta u)],
$$

where $\Delta u$ is the potential energy difference resulting from the displacement, and $c$ is a positive constant which, for a macroscopic system, is related to its temperature. In the present case, it is not necessary to exactly specify $c$. We would rather prefer to regard $c \Delta u$ as a dimensionless potential energy difference. When the interaction between the molecule and the pore surfaces are described using a potential equation (Eq. 4), we no longer need the parameter $p$ defined above since the probability of adsorption is determined by $\epsilon$ through $p_{\text {displacement }}$ defined above. As described previously, in order to account for the adsorption/desorption rate relative to the random diffusive flux, we have to introduce the parameter $m$, which is the number of units of time an adsorbed molecule stays adsorbed before a desorption attempt is made. Since, in dealing with 
asphaltene molecules one has to deal with aggregation-disaggregation phenomena, these must also be accounted for in the simulation. Once a large molecule like an asphaltene delaminates and fragments into several pieces, the motion of each fragment must then be followed, accounting for the fact that the fragments can join together again (similar to aggregation of colloidal particles) at a later time.

\section{B.3.4. Molecular Dynamics Simulations}

The DMCM described above will give a satisfactory insight into the fundamental processes of asphaltene diffusion. The MC method, however, has one major disadvantage. Real time is not an explicit parameter of the simulation. MC methods are, therefore, strictly not applicable to the study of highly non-equilibrium diffusion processes. Molecular dynamics (MD) calculations on the other hand circumvent this difficulty. $\mathrm{MD}$ is a simulation technique in which the equations of motion for a system are directly solved to determine the time evolution of the system. The solution obtained in a MD study is essentially exact to the extent that the potential model used accurately describes the intermolecular forces. MD methods are now fairly well known and used for studying equilibrium properties and one-particle dynamic properties such as self diffusion coefficients. They have been widely used [55] to study simple fluids, nonpolar polyatomics, polar molecules, and bipolymers.

The feasibility of calculating accurate $D_{e}$ with MD simulations was demonstrated by Jacucci and McDonald [56], Jolly and Bearman [57] and Sihoen and Hoheisel [58]. Stoker and Rowley [59] used the techniques developed in these previous studies to simulate binary mixtures of polyatomic molecules. Tirrell, Davis and co-workers [60] have used MD simulations to study diffusion of simple molecules in a single pore. Either equilibrium or nonequilibrium MD can be used to estimate diffusion coefficients. In view of the structural complexities of the porous medium and the molecules in our study, we shall only employ equilibrium MD simulations. One expects the qualitative trends given by the two methods to be similar. The long-time tail in equilibrium simulations can be accounted for by appropriately long simulations. The purpose of the MD simulations here is twofold: first, to compare $D_{e}$ evaluated using the mean-squared displacement methods [Eq. (2)] (from both MC and MD) and those obtained using velocity autocorrelation functions,

$$
D_{e f f}=\frac{1}{3} \int_{0}^{\infty}\langle v(0) \cdot v(\tau)\rangle d t
$$

where $v(0)$ and $v(\tau)$ are velocities of a molecule at times zero and $\tau$ respectively, and $\langle\ldots\rangle$ indicates an average over sufficiently large number of molecules and for various choices of zero times. Secondly, in the case of diffusion with adsorption, a comparison between MC and MD can be used to establish the significance of and, if possible, the appropriate quantitative magnitude of the parameter $m$. 
The study by Schoen and Hoheisel [58] indicated that long runs are required to accurately calculate the velocity correlation function. Of prime importance in improving the statistics of the calculations are the number of time origins used in the correlation function determination, the number of particles, and the length over which the integration is carried out. Despite its rigor, MD still remains a very promising but underdeveloped approach to the determination of transport properties of real molecules in practical systems. To the best of our knowledge, a large scale MD calculation has never been attempted for a complex pore space and molecular structures, such as those that in this study. The only drawback of MD is that for complex molecular structures such as those of asphaltenes, the intramolecular and intermolecular potentials are not exactly known. One way of attacking this problem is to use the most accurate and plausible potentials and to adjust the parameters potentials to match the experimental data.

Before the full potential of MD simulation for these system can be realized, eventually several problems must be overcome: (1) It is possible that for some systems the diffusional time scale and that for adsorption/desorption are very different so that during the course of a normal simulation of acceptable length, one of these processes cannot be adequately observed; (2) The type of MD simulation described is not a typical MD simulation because the number of molecules in the system $(N)$ is not constant. When more molecules are added to the system, they must be introduced with the appropriate accelerations, velocities and intermolecular potential energies. These quantities must be appropriately preselected so as not to introduce arbitrary variables or undesirable large transients; and (3) The Green-Kubo expressions for the diffusivity [Eq. (6)] are derived for homogeneous systems. Their applicability to porous media has not been established. Our studies will hopefully provide definitive answers to some of these questions.

\section{RESEARCH COMPLETED THIS QUARTER}

The following were accomplished this quarter:

- During this quarter two papers were submitted to the AIChE Journal. The first paper reports work relevant to task B.3.4. In this system we study the diffusivity of Lennard-Jones particles in porous systems with dimensionality between two and three. A model porous system of such dimensionality is pillared clays. In our simulations the pillared clays were represented by parallel sheets separated by a given distance, and connected by pillars of a given size. Two different spatial distributions of the pillars, namely, random and uniform, were studied to determine their effect on the properties of the system. Our calculations did not indicate a strong dependence of the diffusivity on the spatial distribution of the pillars, except at low porosities. The solvation force was found to be monotonically increasing with decreasing 
porosity of the clays and increasing density of the molecules. Percolation threshold of the system, i.e., the critical porosity, $\varphi_{c}$ below which no macroscopic diffusion is possible, was estimated from the diffusivity measurements in the limit of infinitely low sorbate densities. We found that near $\varphi_{c}$ the diffusivity $D_{l}$ vanishes according to the power law, $D_{l} \propto\left(\varphi-\varphi_{c}\right)^{n}$, where $\varphi$ is the porosity of the system, and $n$ is an universal constant.

- The second paper reports on work which was initiated during the first quarter and briefly discussed in quarterly report \#2. It concerns itself with the sorption and aggregation of asphaltene particles with porous media such as catalysts. It is also of more general interest to those working in the area of oil reservoirs. In this paper we present extensive new experimental data for the amount of asphalt precipitation formed with various solvents. We then use a thermodynamic model based on the Flory-Huggins theory of polymer solutions, together with the Soave equation of state, to predict the data. The model is critically evaluated, and it is shown that its predictions are generally not in good agreement with our data. As an alternative, we propose a new model that employs a scaling equation, somewhat similar to those encountered in aggregation and gelation phenomena. The scaling function takes on a very simple form, and its predictions are in very good agreement with our data. It also predicts that the onset of deposition obeys a simple universal equation.

- Our high pressure diffusion apparatus has been modified and it can new hold up to four tubular membranes. The rejection cofficients of a number of model solvents and solutes has been measured. Results of these studies will be reported during the 6th quarter at the MRS meeting. 


\section{LITERATURE REFERENCES}

1. F. M. Dautzenberg, J. Van Klinken, K. M. A. Pronk, S. T. Sie and J. B. Wiffels, ACS Symp. Ser., 55, pp. 254-267, Washington, D.C. (1978); R. B. Galbreath and R. P. Van Driesen, Proc. 8th World Petrol. Congr., 4, 129 (1971); D. C. Green and D. H. Broderick, Chem. Eng. Prog., 77, 33 (1981); Y. T. Shah and J. A. Paraskos, Ind. Eng. Chem. Proc. Des. Dev., 14, 368 (1975).

2. J. C. Spry and W. H.Sawyer, paper presented at the AIChE Annual Meeting, Los Angeles (1975).

3. J. L. Anderson and J. A. Quinn, Biophys. J., 14, 130 (1979).

4. M. Inoguchi, Sokubai, 18, 78 (1976).

5. A. S. Eigenson, Y. A. Zaitova, G. A. Berg, L. I. Volkova, N. A. Kochetova, T. P. Anmdryshenko, L. Y. Shudina and A. A. Ioakimis, Int. Chem. Eng., 9, 332 (1977).

6. C. Philippopoulos and N. Papayannakos, Ind. Eng. Chem. Res., 27, 415 (1988).

7. M. Ternan, Can. J. Chem. Eng., 61, 689 (1983); A. H. Hardin, R. H. Packwood and M. Ternan, Prep. Div. Petrol. Chem. Am. Chem. Soc., 23(4), 1450 (1978; A. H. Hardin and M. Ternan, Prep. 2nd World Congr. on Chem. Eng., 3, 134 (1981).

8. L. M. Polinski, G. J. Steigel and L. Saroff, Ind. Eng. Chem. Proc. Res. Dev., 21(3), 477 (1982); G. J. Steigel, L. M. Polinski and R. E. Tischer, Ind. Eng. Chem. Proc. Res. Dev., 21(3), 477 (1982).

9. M. C. Scooter and B. L. Crynes, Ind. Eng. Chem. Proc. Res. Dev., 14, 199 (1975).

10. Y. K. Yen, D. E. Furlani and S. W. Weller, Ind. Engng. Chem. Prod. Res. Dev., 15, 24 (1976).

11. J. A. Brooks, R. J. Bertolacini, L. C. Gutbertlet and D. K. Kim, EPRI Publication, AF-160 (1976); R. J. Bertolacini, L. C. Gutherlet, D. K. Kim and K. K. Robinson, EPRI Final Report AF 1084, June, 1979; D. K. Kim, R. J. Bertolacini, J. M. Forgac, R. J. Pellet, K. K. Robinson and C. V. McDaniel, EPRI Final Report AF 1233, November 1979.

12. R. L. McCormick, J. A. King, T. R. King and H. W. Haynes, Ind. Eng. Chem. Res., 28, 940 (1989). 
13. L. Berg and N. K. Kim, Chem. Eng. Commun., 32, 391 (1985).

14. M. C. Tsai and E. Ruckenstein, AIChE J., 27, 697 (1981).

15. P. E. Savage and M. Javanmardian, Ind. Eng. Chem. Res., 28, 381 (1989); K. S. Tsakalis, T. T. Tsotsis and G. J. Steigel, J. Catal., 88, 188 (1984).

16. W. M. Deen, AIChE J., 33(9), 1409 (1987).

17. J. R. Peppenheimer, E. M. Renkin and L. M. Borrero, Am. J. Physiol., 13, 167 (1951); E. M. Renkin, J. Gen. Physiol., 38, 225 (1954).

18. J. D. Ferry, J. Gen. Physiol., 20, 95 (1936).

19. H. Brenner and L. J. Gaydos, J. Coll. Int. Sci., 43, 312 (1977); H. Brenner, Physicochem. Hydro., 1, 91 (1980); L. C. Nitsche and H. Brenner, to be published.

20. R. Aris, Proc. Roy. Soc. Lond., A235, 67 (1941); G. I. Taylor, Proc. Roy. Soc. Lond., A219, 186 (1953).

21. E. A. Dimarzio and C. M. Guttman, Polym. Lett., 7, 131 (1970); J. Chromatogr., 55, 83 (1971); D. G. Leuitt, Biophys. J., 15, 533 (1975); E. N. Lightfoot, J. D Bassingthwaite and E.F. Babrowski, Biophys. J., 9, 167 (1977).

22. C. N. Satterfield, C. K. Colton and W. H. Pitcher, AIChE J., 19, 478 (1973).

23. B. D. Prasher and Y. H. Ma, AIChE J., 23, 303 (1977); B. D. Prasher, G. A. Gabriel and Y. H. Ma, AIChE J., 24, 1118 (1978).

24. C. K. Colton, C. N. Satterfield and C. J. Lai, AIChE J., 21, 289 (1975).

25. D. S. Cannel and F. Rondelez, Macromolecules, 13, 1449 (1950).

26. P. B. Price and R. M. Walker, J. Appl. Phys., 33, 3400 (1947); 33, 3407 (1947).

27. C. P. Bean, M. V. Doyle and G. J. Entine, J. Appl. Phys., 41, 1454 (1970); J. A. Quinn, J. L. Anderson, W. H. Ho and W. J. Petzny, Biophys. J., 12, 990 (1972); R. E. Beck and J. S. Schultz, Biochim. Biophys. Acta, 255, 273 (1972); D. M. Malone and J. L. Anderson, AIChE J., 23, 97 (1977).

28. R. J. Thrash and R. H. Pildes, ACS Preprints, Div. Pet. Chem., 26(2), 515 (1981). 
29. M. Shimura, Y. Shiroto and C. Takeuchi, paper presented at the ACS Meeting, Div. Coll. Surf. Chem., Las Vegas, NV (1982).

30. M. Sakai, K. Sasaki and M. Inagaki, Carbon, 21, 593 (1983).

31. K. Jost, W. Steuer and I. Halasz, Chromatographia, 20, 700 (1985).

32. R. L. Mieville, D. M. Trauth and K. K. Robinson, ACS Preprints, Div. Pet. Chem., 34(3), 635 (1989).

33. R. E. Baltus and J. L. Anderson, Chem. Eng. Sci., 38, 1959 (1983); R. E. Baltus, Catalysis on the Energy Scene, S. Kaliagne and A. Mahay, eds., Elsevier, Amsterdam, p. 533 (1984); R. E. Baltus and J. L. Anderson, Fuel, 63, 530 (1984);

34. R. E. Baltus, K. C. Kyriacou, V. V. Sivaramakrishna and P. Rahimi, AIChE Symp. Ser., No. 266, 84, 50 (1988); K. C. Kyriacou, V. V. Sivaramakrishna, R. E. Baltus and P. Rahimi, Fuel, 67, 15 (1988); K. C. Kyriacou, R. E. Baltus and P. Rahimi, Fuel, 67, 109 (1988); R. L. Nortz, R. E. Baltus and P. Rahimi, Ind. Eng. Chem. Res., 29(9), 1968 (1990).

35. J. Garcia de la Torre and V. A. Bloomfield, Biopolymers, 17, 1605 (1978).

36. R. C. Sane, I. A. Webster and T. T. Tsotsis, Stud. Surf. Sci. Catal., 38, 705 (1988); ACS Symp. Ser., Amer. Chem. Soc., 33(3), 237 (1988); AIChE Symp. Ser., 84, 57 (1988); R. C. Sane, I. A. Webster, T. T. Tsotsis and V. S. Ravi Kumar, in press, Asphalt and Asphaltenes, T. F. Yen, ed.; R. C. Sane, I. A. Webster, T. T. Tsotsis and V. S. Ravi Kumar, submitted for publication, Chem. Eng. Sci.; R. C. Sane, Ph.D. Thesis, University of Southern California, December, 1990.

37. J. W. Bunger and D. E. Cogswell, Adv. Chem. Ser., "The Chemistry of Asphaltenes," 195, 219 (1981).

38. J. F. McKay, P. J. Amend, T. E. Cogswell, P. M. Harnsberger, R. B. Erikson and D. R. Latham, Adv. Chem. Ser., "Petroleum Asphaltenes: Chemistry and Composition," 170, 128-142 (1978); J. A. Koots and J. G. Speight, Fuel, 54, 179 (1975); I. A. Wiehe, paper presented at the AIChE Spring National Meeting, Orlando, Florida, March 18-22 (1990).

39. R. B. Long, Adv. Chem. Ser., "The Chemistry of Asphaltenes," 195, 17 (1981); R. B. Long and J. G. Speight, Revue de L'Institut Français du Petrolé, 44, 205 (1989).

40. C. E. Snape and K. D. Bartle, Fuel, 63, 883 (1984); 64, 427 (1985). 
41. J. P. Dickie and T. F. Yen, Anal. Chem., 39(14), 1897 (1967).

42. N. Nourbakhsh, I. A. Webster and T. T. Tsotsis, Appl. Catal., 50, 65 (1989); N. Nourbakhsh, A. Champagnie, I. A. Webster and T. T. Tsotsis, AIChE Symp. Ser., 85, 75 (1989); N. Nourbakhsh, B. J. Smith, I. A. Webster, J. Wei and T. T. Tsotsis, J. Catal., 27, 178 (1991).

43. D. L. Cocke, E. D. Johnson and R. F. Merrill, Cat. Rev. Sci. Eng., 26, 148 (1984); L. Young, Anodic Oxide Films, Academic Press, New York (1961); K. Itaya, S. Sugawara, K. Arai and S. Saito, J. Chem. Eng. Japan, 17(s), 514 (1984).

44. A. M. Champagnie, T. T. Tsotsis, R. G. Minet and I. A. Webster, Chem. Eng. Sci., 45(6), $1443(1990)$.

45. W. T. Mo and J. Wei, Chem. Eng. Sci., 41, 4, 703 (1986); N. Otani and J. M. Smith, J. Catal., 5, 332 (1966).

46. D. Ryan, R. G. Carbonell and S. Whitaker, Chem. Eng. Sci., 35, 10 (1980).

47. T. T. Tsotsis, R. Sane, I. A. Webster and J. D. Goddard, J. Catal., 101, 416 (1986); J. D. Goddard, T. T. Tsotsis and M. N. Kassem, paper presented at the AIChE Annual Meeting, Miami, Florida, November (1986).

48. J. S. Schultz, J. D. Goddard and S. R. Suchdeo, AIChE J., 20, 625 (1974); J. D. Goddard, Chem. Eng. Sci., 32, 795 (1977).

49. J. D. Goddard, in Transport With Chemical Reaction, P. Stroeve and W. J. Wards, eds., AIChE Symp. Ser., 202, 114 (1982).

50. M. Sahimi, G. R. Gavalas and T. T. Tsotsis, Chem. Eng. Sci., 45, 1443 (1990).

51. P. L. Paine and P. Scherr, Biophys. J., 15, 1087 (1975)).

52. M. Sahimi and A. O. Imdakm, J. Phys. A, 21, 3833 (1988); Phys. Rev. Lett., 66, 1169 (1991); Chem. Eng. Sci., 46, 1977 (1991); M. Sahimi and V. L. Jue, Phys. Rev. Lett., 62, 629 (1989).

53. M. Sahimi, J. Chem. Phys., 92, 5107 (1990).

54. D. Fincham, N. Quirke and D. J. Tildeshy, J. Chem. Phys., 84, 4553 (1986).

55. M. P. Allen and D. J. Tildeshy, Computer Simulations of Liquids, (Clarendon, Oxford, 1987); D. J. Adams , E. M. Adams, and G. J. Hills, Mol. Phys., 38, 387 (1979); S. Murad, A. 
Papaioannou and J. G. Powler, Mol. Phys., 56, 431 (1985); P. J. Rossky, Ann. Rev. Phys. Chem., 36, 321 (1985).

56. G. Jacucci and I. R. McDonald, Physica, 80A, 607 (1975).

57. K. L. Jolly and R. J. Bearman, Mol. Phys., 41, 137 (1980).

58. M. Schoen and C. Hohiesel, Mol. Phys., 52, 33 (1984); 52, 1024 (1984).

59. J. M. Stoker and R. L. Rowley, J. Chem. Phys., 91, (1989).

60. J. J. Magda, M. V. Tirrell and H. T. Davis, J. Chem. Phys., 83, 1888 (1985); 85, 6674 (1986); I. Bitsanis, J. J. Magda, M. V. Tirrell and H. T. Davis, J. Chem. Phys., 87, 1733 (1987). 

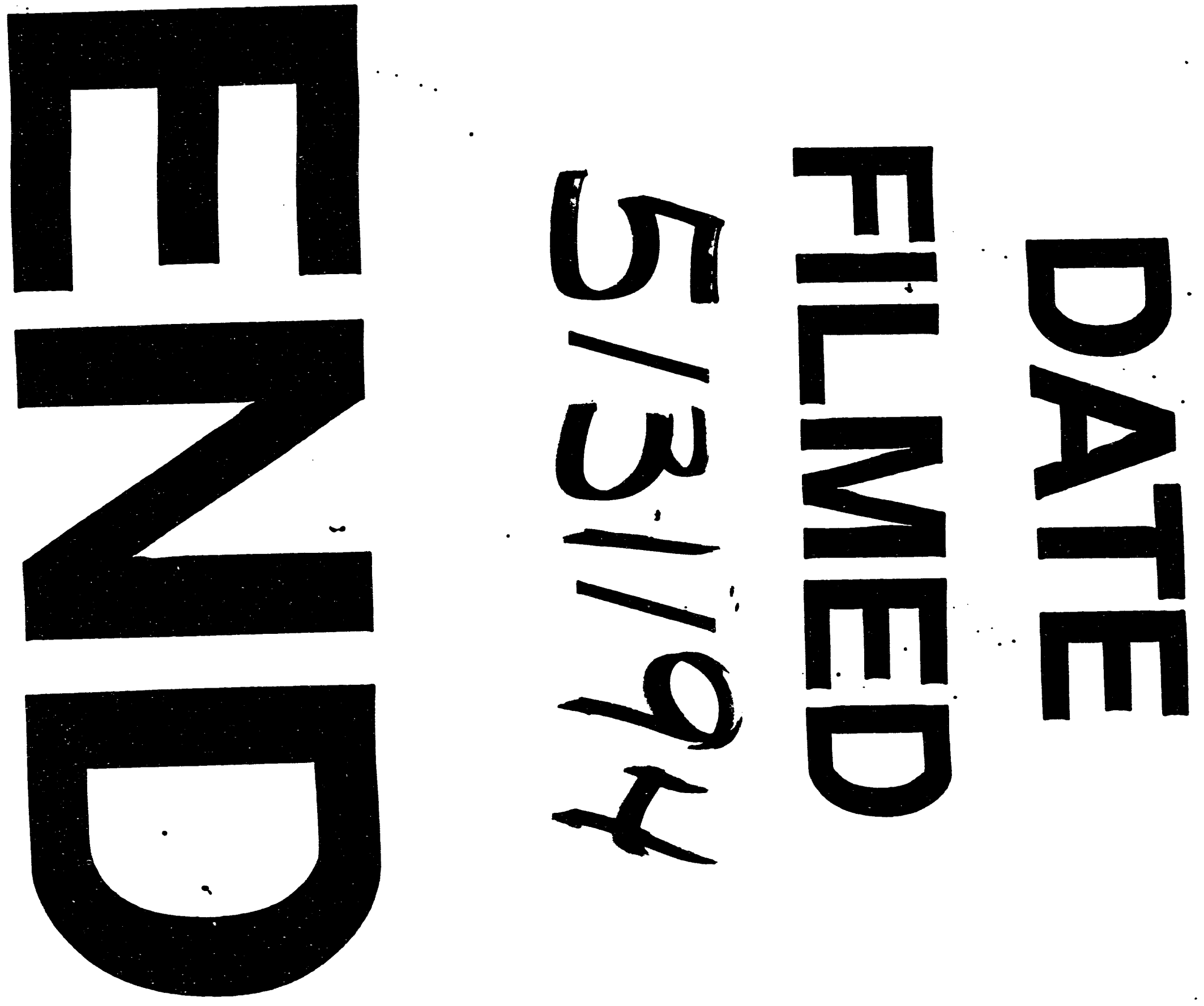

$x$ 

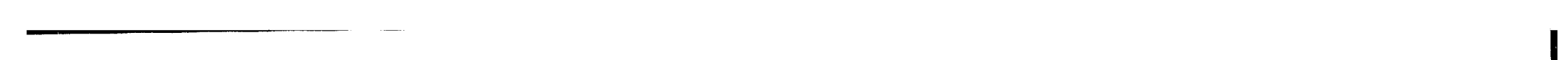

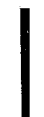

